\title{
Basaloid carcinoma of the lung associated with central cavitation: a unique surgical case focusing on cytological and immunohistochemical findings
}

\author{
Sohsuke Yamada ${ }^{*}$, Hirotsugu Noguchi ${ }^{1}$, Atsunori Nabeshima ${ }^{1}$, Takashi Tasaki ${ }^{1}$, Shohei Kitada ${ }^{1}$, Tetsuro Baba ${ }^{2}$,
} Hidetaka Uramoto ${ }^{2}$, Takeshi Hanagiri ${ }^{2}$ and Yasuyuki Sasaguri ${ }^{1}$

\begin{abstract}
A history of an increase in pulmonary mass was presented in the right upper lobe of a 72-year-old male. The bronchial brushing cytology specimens contained many sheet-like or three-dimensional clusters of malignant cells having small to medium-sized, uniform oval to round, and hyperchromatic nuclei, inconspicuous nucleoli, and scanty cytoplasm, admixed with mitotic figures. A coarsely granular chromatin pattern was predominantly noted. We first interpreted it as suspicious of malignancy, such as atypical carcinoid. A right upper lobectomy was performed, and gross examination revealed a centrally cavity-formed tumor lesion, containing asymmetrically thinned wall and looking grayish to whitish, partly adjacent to the bronchiolar wall. On microscopic examination, the tumor was predominantly composed of a solid proliferation of atypical epithelial cells without apparent glandular or squamous differentiation, often arranged in an alveolar growth pattern with peripheral palisading. Immunohistochemically, these atypical cells are negative for all three neuroendocrine markers and thyroid transcription factor 1, whereas positive for 34BE12, p63 and S-100 protein. Therefore, we finally made a diagnosis of basaloid carcinoma with cavity formation. We should be aware that, owing to its characteristic features, cytopathologists might be able to raise basaloid carcinoma of the lung as one of differential diagnoses, based on careful cytological examination.
\end{abstract}

Virtual slides: The virtual slide(s) for this article can be found here: http://www.dianosticpathology.diagnomx.eu/vs/ 1519986488570234

Keywords: Basaloid carcinoma, Lung, Cytology, Cavitation

\section{Background}

Although basaloid carcinoma $(\mathrm{BC})$ of the lung is a rare subtype of nonsmall cell lung cancer, one original paper described that up to $32 \%$ tumors were reclassified as $\mathrm{BCs}$ among a large series of surgically resected lung cancers with retrospective review, originally classified as poorly or undifferentiated carcinoma [1]. In 1975, Spencer first reported the histopathologic, immunohistochemical, ultrastructural, and clinical features of BC of the lung [2]. Subsequently, this entity was established based on the following criteria, as previously described by Brambilla et al. in 1992 [3]: (1) A solid lobular or

\footnotetext{
* Correspondence: Sousuke@Med.Uoeh-U.Ac.Jp

${ }^{1}$ Departments Of Pathology And Cell Biology, School Of Medicine, University Of Occupational And Environmental Health, Kitakyushu, Japan Full list of author information is available at the end of the article
}

anastomotic trabecular pattern growing invasively in a finger-like fashion from the bronchial and/or glandular duct lining; (2) Small cuboidal to fusiform cells of mean diameter 12 to $15 \mu \mathrm{m}$, with moderately hyperchromatic nuclei and without prominent nucleoli. There was a scant but visible cytoplasm, and no nuclear molding; (3) Peripheral palisading with radially arranged cells at the periphery of lobules; (4) A high rate of mitosis, between 15 and 44 per 10 high-power fields. The World Health Organization (WHO) classification of tumours of the lung now records carcinoma with basaloid pattern, either as a pure $\mathrm{BC}$, a variant of large cell carcinoma with above typical histopathologic patterns, or as a basaloid variant of squamous cell carcinoma when coexisted with areas of squamous differentiation [4]. BC of the lung often poses a diagnostic challenge to clinicians and

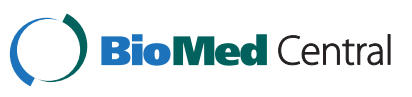


cytopathologists, since its entity is difficult to diagnose pre-operatively $[5,6]$. In fact, one old paper reported that the cytologic differentiation of $\mathrm{BC}$ extensively overlap with those of small cell carcinoma [5]. Although another group demonstrated that patients with $\mathrm{BC}$ of the lung did not have a poor prognosis than the other nonsmall cell lung cancers [7], Brambilla et al. recently have confirmed that lung carcinoma with a basaloid pattern is a unique entity with a significantly worse outcome [8], similar to BCs in organs other than the lungs [3]. Thus, it would be critical to establish an accurate preoperative diagnosis by bronchial brushing and washing cytology.

Indeed, pulmonary BC could be a relatively uncommon disease, but not as compared with some recently published case reports of extremely rare tumor cell types in the lung $[9,10]$. Despite of that, we report a unique surgical case of BC of the lung, associated with central cavitation. Based on the cytology specimens, we preoperatively interpreted it merely as suspicious of carcinoma.

\section{Materials and methods}

The patient was a 72-year-old Japanese man. Bronchial brushing and washing cytology, and transbronchial lung biopsy from the pulmonary mass were performed, followed by a right upper lobectomy. The tumor specimens after fixation in $10 \%$ neutral buffered formalin were embedded in paraffin for histological or immunohistochemical examinations. All immunohistochemical stainings were carried out using Dako Envision kit (Dako Cytomation Co., Glostrup, Denmark) according to the manufacturer's instructions, and using commercially available prediluted monoclonal antibodies against the following antigens: synaptophysin (Dako, diluted 1:20), chromogranin A (Dako, diluted 1:200), CD56 (NICHIREI, diluted 1:1), cytokeratins (34ßE12; Leica Microsystems, Wetzlar, Germany, diluted 1:200, CK7; Dako, diluted 1:50, and CK20; Dako, diluted 1:60), p63 (Dako, diluted 1:30), S-100 protein (Dako, diluted 1:900), thyroid transcription factor 1 (TTF-1; Dako, diluted 1:100), $\alpha$-smooth muscle actin ( $\alpha$-SMA; Dako, diluted 1:150), h-caldesmon (Dako, diluted 1:50), calponin (Dako, diluted 1:50), CD10 (NOVOCASTRA laboratories Ltd., Newcastle, United Kingdom, diluted 1:20), CEA (Dako, diluted 1:50), and Ki-67 (MIB-1; Dako, diluted 1:50). The profile of all these antigens is summarized in Table 1. Since all tumor specimens were fixed in formalin, transmission electron microscopy could not be performed.

\section{Case presentation}

The patient had a history of cerebral infarction 10 years ago. He was a heavy smoker over 50 years. There was no history of malignancy, immunosuppressive disorders,
Table 1 The profile of all immunohistochemical antigens

\begin{tabular}{lll}
\hline Antigens & Sources of antibodies & Dilution \\
\hline 34ßE12 & Leica Microsystems & diluted 1:200 \\
CK7 & Dako & diluted 1:50 \\
CK20 & Dako & diluted 1:60 \\
Chromogranin A & Dako & diluted 1:200 \\
Synaptophysin & Dako & diluted 1:20 \\
CD56 & NICHIREI & diluted 1:1 \\
TTF-1 & Dako & diluted 1:100 \\
CEA & DAko & diluted 1:50 \\
h-caldesmon & Dako & diluted 1:50 \\
S-100 protein & Dako & diluted 1:900 \\
p63 & DAko & diluted 1:30 \\
a-SMA & Dako & diluted 1:150 \\
CD10 & NOVOCASTRA & diluted 1:20 \\
Calponin & Dako & diluted 1:50 \\
\hline
\end{tabular}

use of immunosuppressive medications, or unusual infections.

During a follow-up of his infarction, a chest X-ray showed a mass shadow with central cavity area in the middle region of the right lung 1 and half years before the surgery. The sputum culture examined detected colonies of Mycobacterium Gordonae, however, following that, a recent increase of pulmonary mass was presented. Laboratory data, including blood cell count and chemistry, were almost within normal limits, except for high levels of blood urea nitrogen (BUN; $38 \mathrm{mg} / \mathrm{dL}$ ) and creatinine $(\mathrm{Cr} ; 1.87 \mathrm{mg} / \mathrm{dL})$, manifesting as mild renal dysfunction. Carcinoembryonic antigen (CEA; $4.3 \mathrm{ng} / \mathrm{mL}$ ), squamous cell carcinoma antigen (SCC; $5.3 \mathrm{ng} / \mathrm{mL}$ ), cytokeratin 19 fragment (CYFRA; $5.3 \mathrm{ng} / \mathrm{mL}$ ), neuron specific enolase (NSE; $13.5 \mathrm{ng} / \mathrm{mL}$ ), and pro-gastrinreleasing peptide (pro-GRP; $78.8 \mathrm{pg} / \mathrm{mL}$ ) levels as tumor markers were modestly increased up, but carbohydrate antigen (CA) 19-9 and sialyl Lewis X-i antigen (SLX) levels were within normal limits. A chest CT scan revealed a relatively well-demarcated mass, measuring approximately $37 \times 30 \times 23 \mathrm{~mm}$, associated with central and variably thin-walled cavity formation, in the right upper lobe, S2. CT scans of the head and abdomen disclosed no definite evidence of metastases in the lymph nodes or other organs. The patient had neither recurrence nor metastases of the lung cancer, respectively, however, was dead due to bronchopneumonia at 3 years after the operation.

\section{Pathological findings}

The first bronchial brushing cytology specimens were consisted of many clusters of cohesive and sheet-like or three-dimensional tumor cells and a small number of 
individual tumor cells without necrotic or hemorrhagic backgrounds (Figure 1A). The bronchial washing cytology specimens were relatively inadequate, but very similar to the findings of brushing one. The malignant cells showed small to medium-sized (12 to $20 \mu \mathrm{m}$ in diameter), relatively uniform, and round to oval with mild pleomorphism and had relatively scanty cytoplasm (Figure 1B). Additionally, the nuclei were hyperchromatic, predominantly in a coarsely granular chromatin pattern, and often had inconspicuous nucleoli, but occasionally mitotic figures (Figure 1B). Rosettes were absent, whereas a very small number of malignant squamoid cells was rarely seen. Based on that, we first interpreted it as suspicious of carcinoma, such as atypical carcinoid, and an ordinary right upper lobectomy was performed. On the other hand, the transbronchial lung biopsy specimens from the pulmonary mass were too small to be diagnostic.

On gross examination, the cut surface revealed a centrally cavity-formed, relatively poorly-demarcated, and solid firm mass, measuring $35 \times 27 \times 25 \mathrm{~mm}$, which looked from grayish to whitish in color, partially adjacent to the bronchiole (Figure 2A). This central cavity measured approximately $30 \times 10 \mathrm{~mm}$, but filled with no necrobiotic materials. The background of the lung had no remarkable change, i.e., not emphysematous (Figure 2A). A scanning magnification of it showed that the cancer components, less than $30 \%$ in volume, were surrounded by the cavity and irregularly grew up along the asymmetrically thickened but relatively thin cavity wall, together with extension to the peripheral alveolar wall in a sheet-like fashion (Figure 2B). This tumor lesion was partly adjacent to the bronchiolo-vascular bundle (Figure 2B). These features might indicate a sequential progression from the bronchiole to the surrounding alveolar space. There were no carcinoma in situ components within our thorough investigation.
Microscopic findings showed a solid and sheet-like proliferation of relatively uniform and small to medium-sized atypical epithelial cells having hyperchromatic nuclei and scant eosinophilic cytoplasm, often arranged in an alveolar fashion with peripheral palisading, typical of $\mathrm{BC}$ of the lung (Figure 3A). By contrast, rosettes structures were absent. Apparent keratinization, intercellular bridge, or glandular differentiation was also not evident, and there was not intracytoplasmic mucin with Alcian-Blue staining. On high-power view, mitotic counts were high (more than 15 per $2 \mathrm{~mm}^{2}$ ) (Figure $3 \mathrm{~B}$ ). The carcinoma cells partly involved the adjacent bronchiolar wall but without evidence of vessel permeation. Moreover, although foci of comedo-type tumor necrosis were not recognized within the cancer nests and the central cavity, the cancer-cavity junction sometimes contained coagulative necrosis of pre-existing alveolar wall (Figure 3C). Immunohistochemically, these carcinoma cells were negative for all three neuroendocrine markers, i.e., synaptophysin, chromogranin A and CD56, TTF-1, CEA, CK20, h-caldesmon, $\alpha$-SMA, calponin, and CD10, but specifically positive for 34ßE12 (Figure 4A), CK7 and p63 (Figure 4B). Additionally, one part of tumor nests was positive for S-100 protein (Figure 4C). On the other hand, MIB-1 labeling index was approximately $5 \%$ in the proliferating atypical cells of the cancer nests. All immunohistochemical profile of the carcinoma cells is summarized in Table 2.

Based on all these features, we suggested that these carcinoma cells were not characteristic of neuroendocrine, squamous, glandular, or transitional differentiation, and finally made a diagnosis of $\mathrm{BC}$ of the lung associated with central cavitation. Final pathological stage was determined as pT2aNOM0, stage IB, according to the International Association for Study of Lung Cancer (IASLC) classification [11].
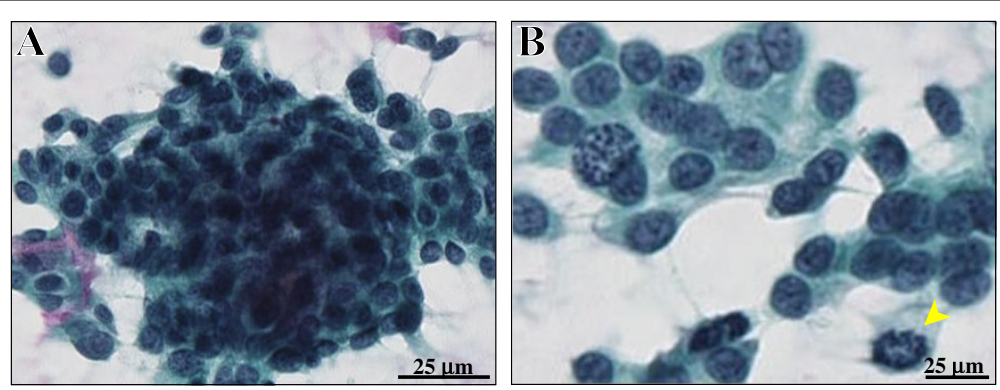

Figure 1 Cytomorphologic examination of the bronchial brushing cytology specimens. (A) The cytology specimens were consisted of many clusters of cohesive and sheet-like or three-dimensional tumor cells and a small number of individual tumor cells without necrotic or hemorrhagic backgrounds (Papanicolaou stains). Bar $=25 \mu \mathrm{m}$. (B) Those malignant cells showed small to medium-sized (12 to $20 \mu \mathrm{m}$ in diameter), relatively uniform, and round to oval with mild pleomorphism and had relatively scanty cytoplasm. Additionally, the nuclei were hyperchromatic, predominantly in a coarsely granular chromatin pattern, and had inconspicuous nucleoli but mitotic figures (arrowhead) (Papanicolaou stains). Rosettes were absent. Bar $=25 \mu \mathrm{m}$. 

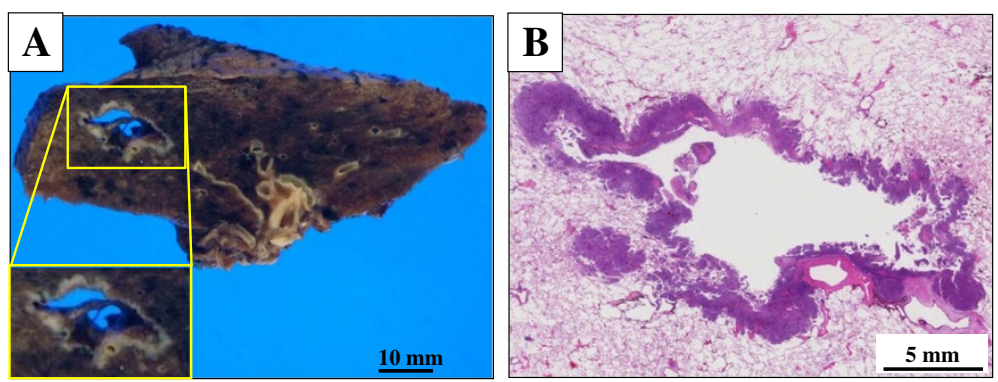

Figure 2 Gross and microscopic examination of the resected specimen. (A) On gross examination, the cut surface revealed a centrally cavity-formed, relatively poorly-demarcated, and solid firm mass, measuring $35 \times 27 \times 25 \mathrm{~mm}$, which looked from grayish to whitish in color, partially adjacent to the bronchiole (lower side, inset). This central cavity measured approximately $30 \times 10 \mathrm{~mm}$, but filled with no necrobiotic materials. Bar $=10 \mathrm{~mm}$. (B) A scanning magnification of it (H\&E stains) showed that the cancer components were surrounded by the cavity and irregularly grew up along the asymmetrically thickened but relatively thin cavity wall, together with extension to the peripheral alveolar wall in a sheet-like fashion (It. side). This tumor lesion was partly adjacent to the bronchiolo-vascular bundle (rt. lower side). There were no carcinoma in situ components in our case. Bar $=5 \mathrm{~mm}$.

\section{Discussion}

Unlike the current BC case, frequent carcinoma in situ components should cause advanced clinical treatment, including more aggressive surgery or adjuvant chemotherapy, even in the early stage for $\mathrm{BC}$ of the lung [8]. It would lead to confer a significantly poor prognosis of $\mathrm{BC}$ amongst nonsmall cell lung cancer in stage I to II patients [8]. Thus, it could be critical to establish an accurate preoperative diagnosis by bronchial brushing and/ or washing cytology, the clinical utility of which in diagnosing pulmonary tumors has been generalized. The cytological characteristic of $\mathrm{BC}$ of the lung would partly reflect the histopathological ones, showing cohesive, three-dimensional and/or sheet-like clusters of relatively small and uniform malignant cells, often having finely granular chromatin, inconspicuous nucleoli, high mitotic rate and scanty cytoplasm, arranged occasionally in a rosette-like pattern, as well as single cells formation in the background of possible necrosis [4-6]. However, in fact, the features of this relatively new and rare entity have not been well described or reviewed more recently. As in the present case, the cytology findings (Figure 1)
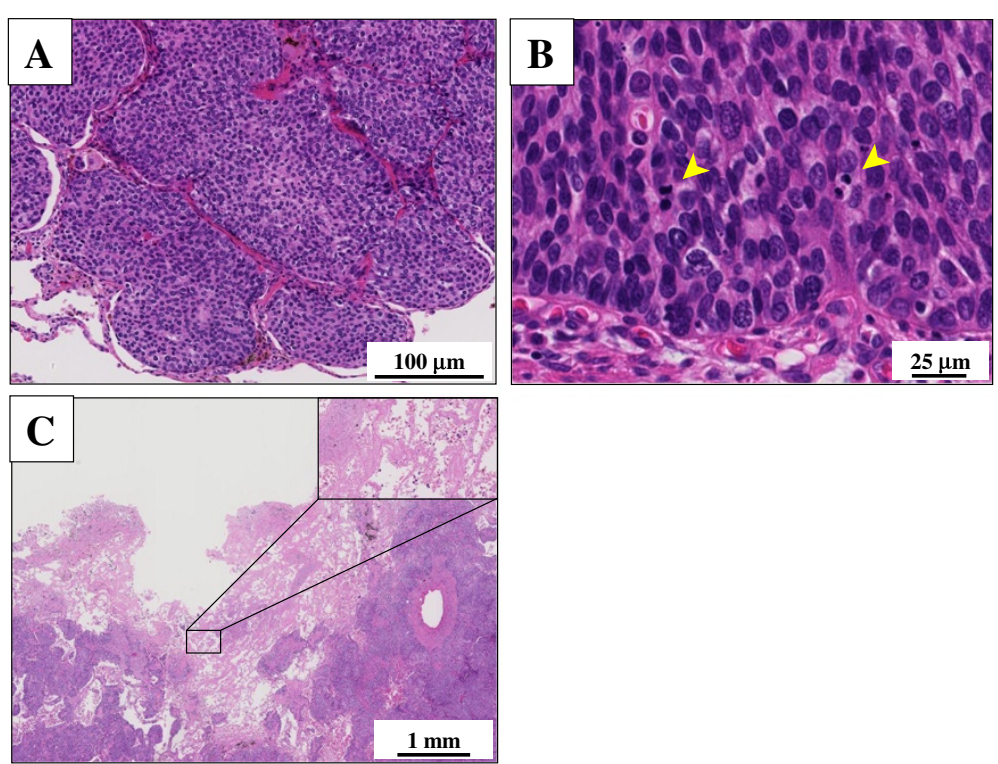

Figure 3 Microscopic examination of the BC of the lung. (A) Low power view showed a solid and sheet-like proliferation of relatively uniform and small to medium-sized atypical epithelial cells having hyperchromatic nuclei and scant eosinophilic cytoplasm, often arranged in an alveolar fashion with peripheral palisading. However, rosettes structures were absent. Apparent keratinization, intercellular bridge, or glandular differentiation was also not evident (H\&E stains). Bar $=100 \mu \mathrm{m}$. (B) On high-power view, mitotic counts (arrowheads) were high (more than 15 per $2 \mathrm{~mm}^{2}$ ) (H\&E stains). Bar $=25 \mu \mathrm{m}$. (C) Although foci of comedo-type tumor necrosis were not recognized within the cancer nests and the central cavity, the cancer-cavity junction contained coagulative necrosis of pre-existing alveolar wall (inset) (H\&E stains). Bar $=1 \mathrm{~mm}$. 

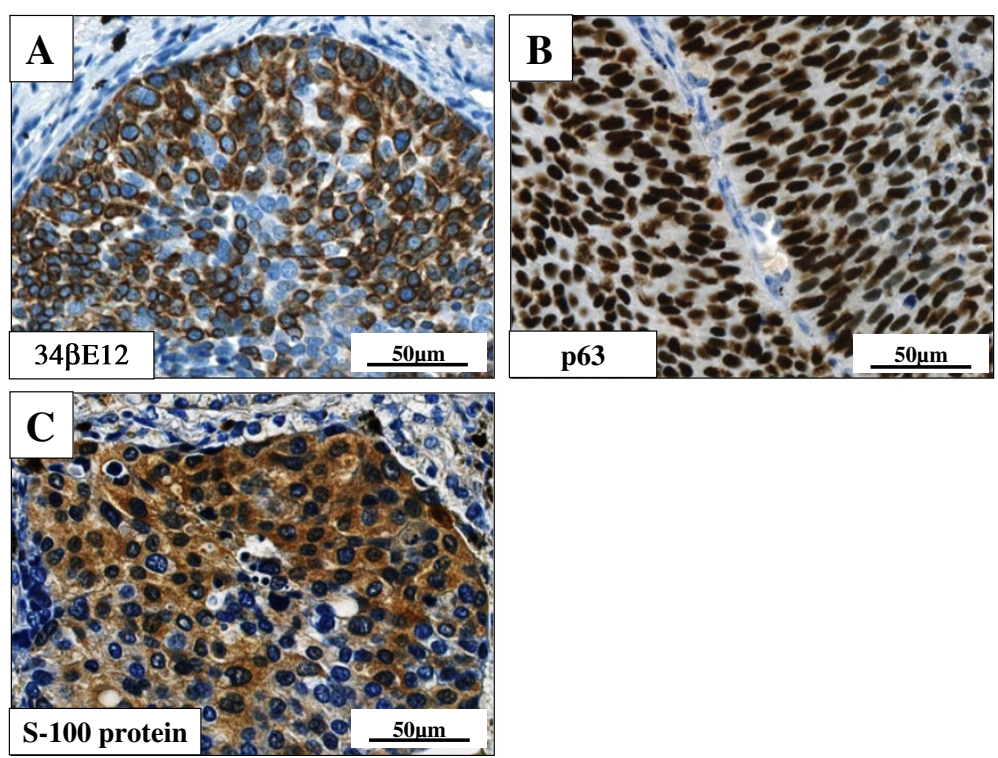

Figure 4 Immunohistochemical examination of the BC of the lung. (A, B, C) The carcinoma cells of BC were specifically positive for $34 \beta E 12$ (A) and p63 (B). Additionally, one part of tumor nests was positive for S-100 protein (C). Bars $=50 \mu \mathrm{m}$.

showed almost similar to those as described above, even though neither rosette-like fashion nor necrotic backgrounds were evident. In spite of that, a confident and accurate diagnosis of $\mathrm{BC}$ of the lung might be impossible only on cytology specimens, likely due to lack of experience, cytomorphologic variety, misinterpretation and/or sampling errors. Nevertheless, in cases without evidence of neuroendocrine or squamous or glandular differentiation, such as ours, cytopathologists should raise possibility of $\mathrm{BC}$ as one of differential diagnoses, other than large cell neuroendocrine carcinoma, atypical carcinoid, small cell carcinoma, poorly differentiated squamous cell carcinoma or adenocarcinoma, or basaloid variant of squamous cell carcinoma, at the very least. Future studies will be further required after collecting and examining a larger number of pulmonary $\mathrm{BC}$ cases.

\section{Table 2 Immunohistochemical profile of the carcinoma} components in our case of $\mathrm{BC}$ of the lung

\begin{tabular}{cc}
\hline positive & negative \\
\hline $34 \beta E 12$ & Chromogranin A \\
CK7 & Synaptophysin \\
S-100 protein & CD56 \\
p63 & CK20 \\
& TTF-1 \\
& CEA \\
& h-caldesmon \\
& a-SMA \\
& CD10 \\
& Calponin \\
\hline
\end{tabular}

It is very likely that our case report is histopathologically remarkable for two reasons at least: first, central cavitation accompanied uniquely within the tissue of $\mathrm{BC}$ (Figure 2). Actually, to date, the number of 'true' cases reported as BC of the lung in the English literatures is not large, and most recent reference is from 2008 within our thorough investigation [8]. According to those papers, all $\mathrm{BC}$ tumors have exhibited nodular or mass lesions without cavity formation, not similar to our case. Furthermore, this case peculiarly showed coagulative necrotic foci of pre-existing alveolar wall in the cancercavity junction (Figure 3C), indicating ischemic change of the pulmonary tissue surrounded by the $\mathrm{BC}$ areas. Xue et al. have very recently proposed that the solitary thin-walled cavity of lung adenocarcinoma would be formed via multiple processes: adenocarcinoma cells initially develop in alveolar wall and grow toward bronchiole, and next formed a unidirectional check-valve owing to lack of cartilage in bronchiole; the accumulations of gases enter alveoli; the alveoli rupture and fuse into cavity with separation; and finally, the cavity gradually gets larger and larger with the increased inner pressure [12]. As in our $\mathrm{BC}$ case, it was suggested that the carcinoma cells firstly developed in the bronchiolar wall and subsequently grew extensively toward alveolar wall, and vice versa, since $\mathrm{BC}$ of the lung could originate from a basal bronchial or bronchiolar epithelial stem cell, as described by Brambilla et al. [3]. In this scenario, the above peculiar histopathological findings (Figure 3C) might confirm their above hypotheses with regard to the pathogenetic mechanisms of solitary thin-walled cavitation in lung cancer [12]. It would be intriguing to study 
this topic after investigating many cases of it. Second, immunohistochemical expression of not merely p63 but S-100 protein was positively seen in the tumor nests (Figure 4). Although there have been no large, detailed immunohistochemical studies of $\mathrm{BC}$ of the lung until now, the results indicate that those tumor cells have potential myoepithelial phenotypes, as well. We could provide the possible evidence for the first time that $\mathrm{BC}$ of the lung might arise from a ductal epithelialmyoepithelial cell, as a result of neoplastic transformation of outer supporting myoepithelial cells, as well as inner ductal epithelial cells [13]. However, since other myoepithelial markers examined, such as $\alpha$-SMA, calponin, and CD10, were negative (Table 2), this suggestion may be highly speculative and partly unsupported. Despite of that, future convincing data will be further required to determine whether our hypothesis is significant or not.

\section{Conclusion}

We herein reported a rare case of $\mathrm{BC}$ of the lung associated with central cavitation. The present case was tentatively diagnosed as suspicious of carcinoma, not otherwise specified, on the cytology specimens, since its features showed unclear differentiation. All cytopathologists should be aware that its cytomorphologically characteristic findings from extensively careful examination might induce one of differential diagnoses, and possibly a correct diagnosis. $\mathrm{BC}$ of the lung may be more common than generally considered.

\section{Consent}

Written informed consent was obtained from the patient for publication of this case report and any accompanying images. A copy of the written consent is available for review by the Editor-in-Chief of this journal.

\section{Competing interests}

The authors declare that they have no competing interests.

\section{Authors' contributions}

SY and HN participated in conception of the idea and writing of the manuscript. SY, HN, TT, AN, SK, TB, HU, TH and YS performed the cytohistological and immunohistochemical interpretation of the tumor tissue. All authors have read and approved the final manuscript.

\section{Author details}

'Departments Of Pathology And Cell Biology, School Of Medicine, University Of Occupational And Environmental Health, Kitakyushu, Japan. ${ }^{2}$ Departments Of Surgery II, School Of Medicine, University Of Occupational And Environmental Health, Kitakyushu, Japan.

Received: 26 November 2012 Accepted: 5 December 2012 Published: 11 December 2012

\section{References}

1. Moro D, Brichon PY, Brambilla E, Veale D, Labat F, Brambilla C: Basaloid bronchial carcinoma. A histologic group with a poor prognosis. Cancer 1994, 73:2374-2739.

2. Spencer H: Pathology of the lung. Oxford: Pergamon Press; 1975.
3. Brambilla E, Moro D, Veale D, Brichon PY, Stoebner P, Paramelle B, Brambilla C: Basal cell (basaloid) carcinoma of the lung: a new morphologic and phenotypic entity with separate prognostic significance. Hum Pathol 1992, 23:993-1003.

4. Travis WD, Colby TV, Corrin B, Shimosato Y, Brambilla E, In collaboration with Sobin LH and pathologists from 14 countries: Large cell carcinoma. In Histological typing of lung and pleural tumours. World health organization. International histological classification of tumors. Edited by Brambilla $\mathrm{E}_{\text {, }}$ Pugatch B, Geisinger K, Gal A, Sheppard MN, Guinee DG, Jiang SX, Lantuejoul S, Chang YL, Peterson I, Meyerson M, Hanash SM, Noguchi M. Berlin: Springer; 1999:45-50.

5. Dugan JM: Cytologic diagnosis of basal cell (basaloid) carcinoma of the lung. A report of two cases. Acta Cytol 1995, 39:539-542.

6. Foroulis CN, lliadis KH, Mauroudis PM, Kosmidis PA: Basaloid carcinoma, a rare primary lung neoplasm: report of a case and review of the literature. Lung Cancer 2002, 35:335-338.

7. Kim DJ, Kim KD, Shin DH, Ro JY, Chung KY: Basaloid carcinoma of the lung: a really dismal histologic variant? Ann Thorac Surg 2003, 76:1833-1837.

8. Moro-Sibilot D, Lantuejoul S, Diab S, Moulai N, Aubert A, Timsit JF, Brambilla C, Brichon PY, Brambilla E: Lung carcinomas with a basaloid pattern: a study of 90 cases focusing on their poor prognosis. Eur Respir J 2008, 31:854-859.

9. Bohn OL, León EA, Lezama O, Rios-Luna NP, Sánchez-Sosa S, LlombartBosch A: Pulmonary artery sarcoma with angiosarcoma phenotype mimicking pleomorphic malignant fibrous histiocytoma: a case report. Diagn Pathol 2012, 7:154.

10. Gong L, Liu XY, Zhang WD, Zhu SJ, Yao L, Han XJ, Lan M, Li YH, Zhang W: Primary pulmonary malignant melanoma: a clinicopathologic study of two cases. Diagn Pathol 2012, 7:123.

11. Groome PA, Bolejack V, Crowley JJ, Kennedy C, Krasnik M, Sobin LH, Goldstraw P, IASLC International Staging Committee; Cancer Research and Biostatistics; Observers to the Committee; Participating Institutions: The IASLC lung cancer staging project: validation of the proposals for revision of the $\mathrm{T}, \mathrm{N}$, and $\mathrm{M}$ descriptors and consequent stage groupings in the forthcoming (seventh) edition of the TNM classification of malignant tumours. J Thorac Oncol 2007, 2:694-705.

12. Xue X, Wang P, Xue Q, Wang N, Zhang L, Sun J, Wang K, Yang B, Wang J: Comparative study of solitary thin-walled cavity lung cancer with computed tomography and pathological findings. Lung Cancer 2012, 78:45-50.

13. Yamada S, Nabeshima A, Tabata T, Guo X, Tasaki T, Wang KY, Shimajiri S, Sasaguri $Y$ : Invasive salivary duct carcinoma ex pleomorphic adenoma of the parotid gland: a teaching case giving rise to the genuine diagnostic difficulty on an inadequate cytology specimen. Diagn Pathol 2012, 7:61.

doi:10.1186/1746-1596-7-175

Cite this article as: Yamada et al:: Basaloid carcinoma of the lung associated with central cavitation: a unique surgical case focusing on cytological and immunohistochemical findings. Diagnostic Pathology 2012 7:175

\section{Submit your next manuscript to BioMed Central and take full advantage of:}

- Convenient online submission

- Thorough peer review

- No space constraints or color figure charges

- Immediate publication on acceptance

- Inclusion in PubMed, CAS, Scopus and Google Scholar

- Research which is freely available for redistribution 